\title{
BMJ Open Immunisation coverage of children in the child welfare system: a systematic review protocol
}

\author{
Jennifer S Hermann, ${ }^{1}$ Robin M Featherstone, ${ }^{2,3}$ Margaret L Russell, ${ }^{4}$ \\ Shannon E MacDonald ${ }^{1}$
}

To cite: Hermann JS, Featherstone RM, Russell ML, et al. Immunisation coverage of children in the child welfare system: a systematic review protocol. BMJ Open 2017;7:e014625. doi:10.1136/ bmjopen-2016-014625

- Prepublication history and additional material are available. To view please visit the journal (http://dx.doi.org/ 10.1136/ bmjopen-2016-014625)

Received 7 October 2016 Revised 9 March 2017 Accepted 22 March 2017

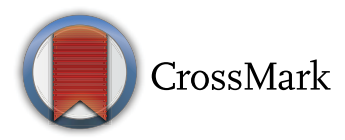

${ }^{1}$ Faculty of Nursing, University of Alberta, Edmonton, Canada ${ }^{2}$ Alberta Research Centre for Health Evidence (ARCHE), University of Alberta, Edmonton, Canada

${ }^{3}$ Knowledge Translation Platform, Alberta SPOR SUPPOR Unit, Edmonton, Canada ${ }^{4}$ Department of Community Health Sciences, Cumming School of Medicine, University of Calgary, Calgary, Canada

Correspondence to Dr Shannon E MacDonald; smacdon@ualberta.ca

\begin{abstract}
Introduction Children may be placed in the care of the child welfare system when they require additional supports or intervention to ensure their safety and security. Transitions in living arrangements (eg, home to foster care and return to home) and other difficult circumstances for these children may result in interruptions in routine preventive healthcare, such as childhood immunisations. The purpose of this systematic literature review is to determine whether immunisation coverage is a problem among children in the child welfare system and identify any known supports and/or barriers to vaccine uptake in this population.
\end{abstract}

Methods and analysis This systematic review will encompass published and unpublished primary research studies that assess $(A)$ immunisation coverage of children in the child welfare system, (B) how this coverage compares to the general population and/or children not in the child welfare system, and (C) supports and barriers affecting immunisation status of these children. Vaccines in the recommended childhood immunisation schedule for each study setting will be considered. Medline, Embase, Cochrane Library, CINAHL, SocINDEX and ERIC will be comprehensively searched. We will also search ProQuest dissertations and theses, the Conference Proceedings Citation Index for Science and Social Science \& Humanities, and a sample of relevant provincial, national and international websites. References of included studies will be manually searched for relevant studies. English language primary studies from 2000 to current focused on immunisations of children (age 0-17 years) in the child welfare system, in a high-income country, will be included. A narrative analysis of key findings from included studies will be performed and presented.

Ethics and dissemination This protocol does not require ethics approval. Planned dissemination includes peerreviewed publication, conference presentations and briefs for policy makers.

Trial registration number This protocol is registered in the PROSPERO International Prospective Register of Systematic Reviews, registration number CRD42016047319.

\section{INTRODUCTION}

Circumstances sometimes arise that prevent parents from providing for the needs of their children. In such circumstances, children
Strengths and limitations of this study

This study will synthesise information to help assess and guide policy.

- This study is systematic and transparent in its approach, as per PRISMA-P guidelines.

- There may be language bias as only studies published in English will be included.

- Study heterogeneity will likely pose challenges for study comparisons.

may require additional supports and intervention from agencies to ensure their safety and care. ${ }^{12}$ This child welfare intervention may take varying forms, from monitoring and supporting the child in the care of their parent or guardian, to 'out-of-home care', that is removal from the home and being placed in foster care, kinship care or residential care. 'Child welfare' is a term that describes various supports or interventions by governmental or private agencies that occur to promote family stability and protect the safety of the child. ${ }^{3}$ The main focus of child welfare is to protect children from abuse and neglect. ${ }^{3}$ Children in the child welfare system who are receiving interventions, specifically those receiving out-of-home care, have been identified as a vulnerable group ${ }^{45}$ with high rates of healthcare needs ${ }^{6}$ and greater susceptibility to poor health outcomes. ${ }^{7}$ Members of this vulnerable population often cope with developmental, physical and psychoemotional challenges. ${ }^{12-9}$ Facilitating healthcare access and improving coordination to appropriate health services play a key role in ensuring positive health outcomes in this at-risk population. ${ }^{68}$ Transitions in living arrangements (eg, home to out-of-home care and return to home) and other difficult circumstances for these children may result in minimal or interrupted use of routine preventive healthcare. $^{61011}$ 
For a vulnerable group with numerous potential health challenges, immunisations are one preventive health measure that lays a foundation for success through prevention of infectious diseases. ${ }^{12}$ Beyond reducing infectious disease burden and death, the WHO promotes immunisations as key to reducing disability and inequity throughout the world. ${ }^{13}$ These larger benefits that result from absence of disease include improved lifetime capability and improved educational attainment. ${ }^{14}$

In order to assess the evidence about whether children in care of the child welfare system receive appropriate immunisations, this systematic review will synthesise the research literature regarding routine immunisation coverage of children in the child welfare system. For the purpose of this review, 'routine immunisations' are those vaccines in the recommended immunisation schedule in each study setting (as identified by the authors), and 'immunisation coverage' is the proportion of eligible children in the study population who received the vaccine(s) being investigated. This work will identify if these children are receiving the necessary immunisations to support healthy growth and development. Understanding the immunisation needs of children in the child welfare system will also inform policy regarding care for these children. Further, this review provides the foundation for future work to assess immunisation coverage in this population.

This protocol development has been guided by the PRISMA-P guidelines ${ }^{15} 16$ and is registered with PROSPERO (CRD42016047319).

\section{Objective}

The objectives of this systematic review are to identify the level of immunisation coverage of routine vaccines among children in the child welfare system and to understand barriers and supports for immunisation in this population. We will focus on high-income countries (as identified by the World Bank's historical classification by income ${ }^{17}$ for each study's year of publication), given that the social welfare context may differ greatly between high-income versus medium-income, and low-income countries.

\section{METHODS AND ANALYSIS}

\section{Studies for consideration}

Types of studies and inclusion criteria

Literature to be considered in this review are published and unpublished reports of primary research, including peer-reviewed journal articles and grey literature (theses, dissertations, government reports, programme evaluations and quality assurance studies). Both experimental and observational study designs will be included, encompassing quantitative, qualitative and mixed methods research. Review articles, conference proceedings, news items, abstracts, poster presentations, editorials and letters to the editor will be excluded. Studies will be included if they meet the following Population, Intervention, Comparison and Outcome (PICO) inclusion criteria.

\section{Population}

The population of interest is all children from birth up to and including 17 years of age who have had involvement with the child welfare system. For the purpose of this review, child welfare intervention is considered to be supports or interventions by governmental or private agencies that occur to promote family stability and protect safety of the child. ${ }^{3}$

\section{Interventions}

The intervention of interest is routine immunisation, as defined earlier.

\section{Comparison group}

Studies with and without comparison groups will be included.

\section{Outcome measures}

The primary outcome of this systematic review is immunisation coverage (as defined earlier). We are interested in immunisation coverage in children with child welfare intervention, and, where available, how this coverage compares to that in the general population or children not in care. The secondary outcome of interest is the barriers and supports affecting immunisation coverage of children in the child welfare system.

\section{Search methods for identification of studies}

The literature search strategy was informed by the research teams' knowledge of the field and a preliminary search of the literature. We analysed subject headings, titles and abstracts for 13 preidentified target articles, and tested these against our search strategy. The final search strategy was created in collaboration with a health research librarian with expertise in systematic review searching.

The search strategy for peer-reviewed literature was developed in MEDLINE (see online supplementary appendix A) and will be adapted to the other databases by the librarian to account for varying syntax and subject headings. The search is to be conducted in Ovid MEDLINE (1946 to present), Ovid Embase (1996 to 2016 week 30), Wiley Cochrane Library (inception to current), CINAHL via EBSCOhost (1937 to the present), SocINDEX via EBSCOhost (1908 to the present) and Ovid ERIC (1965 to February 2016). We will include English language studies published in 2000 or later, given that child welfare and health service delivery programmes have changed over time. Database limits will be applied to filter out conference proceedings, news items, editorials and letters to the editor from results.

Grey literature sources will be searched to account for any potential publication bias. We will search government reports, theses, dissertations, programme evaluation and quality assurance papers from key provincial, national 
and international agencies and associations. We will also search two conference proceedings databases to identify additional non-peer-reviewed reports: Conference Proceedings Citation Index-Science (CPCI-S) and Conference Proceedings Citation Index-Social Science \& Humanities (CPCI-SSH). We will run searches in Google Scholar to determine if full reports of the proceedings have been published, and if no report is found, the authors will be contacted. Reference lists of included studies will be checked for relevant literature that meets inclusion criteria.

\section{Data collection and analysis}

\section{Selection of studies}

Two independent reviewers will determine whether each study meets the inclusion criteria. An initial screening of titles and abstracts against the predetermined inclusion criteria will occur. Articles that are included after title and abstract screening will undergo full text screening against the inclusion criteria. Decisions of the two reviewers will be compared to ensure consistency in screening and any discrepancies that are not negotiable between the two individuals will be resolved by the principal investigator.

To ensure rigour and a transparent process, the selection process and characteristics of excluded studies will be documented in a PRISMA flow chart. ${ }^{18}$ Excel and EndNote will be used to manage the literature and review process.

\section{Data extraction and management}

Two independent reviewers will extract data from the included literature using a data extraction table in Excel; the study details to be extracted are listed in online supplementary appendix B. The draft data extraction form will be piloted and revised prior to full data extraction. Information to be extracted includes general information about the publication (eg, country, date of publication, details of funding); design, methods and data collection; population; interventions; and data analysis and results. Outcomes will be collected as reported in the original study. If disagreement arises during data extraction, the principal investigator will be consulted. Correspondence to authors will occur for any missing data.

\section{Methodological quality and risk of bias assessment}

To assess risk of bias, the Newcastle-Ottawa Scale (NOS) ${ }^{19}$ will be used for case control and cohort studies. The adapted NOS will be used for cross-sectional studies. ${ }^{20}$ For qualitative and mixed method studies, the Mixed Methods Appraisal Tool ${ }^{21}$ will be used. Each of these tools awards points for studies that include a comparison group, meaning that such studies will receive a higher quality appraisal score. Two independent researchers will apply the relevant assessment tool to all included studies and will document justification of their judgements of risk of bias. If discrepancies occur, discussion will occur until consensus is reached. Unresolved discrepancies by these methods will be resolved by the principal investigator.
Data analysis and synthesis

The review will be reported according to PRISMA guidelines. ${ }^{18}{ }^{22}$ We will describe all studies that meet our inclusion and exclusion criteria, but only include medium and high quality studies in our synthesis. Based on an initial search of relevant studies, we anticipate that heterogeneity of study design, participants, and/or outcomes will preclude meta-analysis of findings. Thus, a narrative synthesis will be completed and synthesis tables will be included. General trends in immunisation coverage will be reported numerically and overarching themes and subcategories of barriers and supports to immunisation will be reported. Given that healthcare provision varies by context, we will analyse coverage, barriers and supports in relation to country, time in care and category of care provision. We also anticipate synthesising vaccine coverage according to varying categories such as multidose vaccines versus single-dose vaccines, and infant-series vaccines versus preschool versus adolescent vaccines. Other categories that emerge in analysis will be included. If protocol amendments are needed, the date of each amendment will be documented along with the change made and the rationale.

\section{ETHICS AND DISSEMINATION}

This review does not require ethics approval as it does not involve contact with human subjects. Planned dissemination includes peer-reviewed publication, conference presentations and briefs for policy-makers.

\section{CONCLUSION}

There is no previous synthesis of the literature on immunisation status, or the barriers/supports to immunisation, for children in care of the child welfare system. Better understanding of how immunisation status in this group compares to that in the general population, and the barriers and supports to immunisation, can support initiatives for addressing deficiencies in health service use by this vulnerable population.

Acknowledgements The authors thank the Knowledge Translation Platform, Alberta SPOR SUPPORT Unit and Dr Meghan Sebastianski for their methodological support of this project, including advice on tools and research librarian support. The authors also thank Krina Rey for her contributions to developing the search strategy.

Contributors SEM conceived and designed the study, participated in developing the search strategy, provided substantial input on drafting the manuscript and approved the final version. JSH contributed to the design of the study, participated in developing the search strategy, drafted the protocol manuscript and approved the final version. RMF guided the development of the search strategy, adapted the search strategy for all databases, critically appraised the manuscript and approved the final version. MR contributed to study design, critically reviewed the manuscript and approved the final version.

Funding This research is supported by a research agreement between the University of Calgary and the Alberta Ministry of Health Research Agreement RSO 1026380. The funding source had no role in study design, collection, analysis or interpretation of data, report writing or publicationdecision.

Competing interests None declared.

Provenance and peer review Not commissioned; externally peer reviewed. 
Data sharing statement Literature search strategies, screening forms, and data extraction forms are available are available from the corresponding author.

Open Access This is an Open Access article distributed in accordance with the Creative Commons Attribution Non Commercial (CC BY-NC 4.0) license, which permits others to distribute, remix, adapt, build upon this work non-commercially, and license their derivative works on different terms, provided the original work is properly cited and the use is non-commercial. See: http://creativecommons.org/ licenses/by-nc/4.0/

(c) Article author(s) (or their employer(s) unless otherwise stated in the text of the article) 2017. All rights reserved. No commercial use is permitted unless otherwise expressly granted.

\section{REFERENCES}

1. Rodrigues VC. Health of children looked after by the local authorities. Public Health 2004;118:370-6.

2. Raman S, Reynolds S, Khan R. Addressing the well-being of aboriginal children in out-of-home care: are we there yet? $\mathrm{J}$ Paediatr Child Health 2011:47:806-11.

3. Canadian Child Welfare Research Portal. Frequently asked questions. http://cwrp.ca/faqs (accessed 25 Aug 2016).

4. Bruskas D. Children in foster care: a vulnerable population at risk. $J$ Child Adolesc Psychiatr Nurs 2008;21:70-7.

5. Zwi K, Joshua P, Moran P, et al. Prioritizing vulnerable children: strategies to address inequity. Child Care Health Dev 2015;41:827-35.

6. Szilagyi MA, Rosen DS, Rubin D, et al. Health care issues for children and adolescents in foster care and kinship care. Pediatrics 2015;136:e1142-66.

7. Leslie LK, Gordon JN, Meneken L, et al. The physical, developmental, and mental health needs of young children in child welfare by initial placement type. J Dev Behav Pediatr 2005;26:177-85.

8. Schneiderman JU, Leslie LK, Arnold-Clark JS, et al. Pediatric health assessments of young children in child welfare by placement type. Child Abuse Negl 2011;35:29-39.
9. Ringeisen $\mathrm{H}$, Casanueva $\mathrm{C}$, Urato M, et al. Special health care needs among children in the child welfare system. Pediatrics 2008;122:e232-41.

10. Nathanson D, Tzioumi D. Health needs of Australian children living in out-of-home care. J Paediatr Child Health 2007;43:695-9.

11. Barnes P, Price L, Maddocks A, et al. Immunisation status in the public care system: a comparative study. Vaccine 2005;23:2820-3.

12. Ehreth J. The global value of vaccination. Vaccine 2003;21:596-600.

13. Andre FE, Booy R, Bock HL, et al. Vaccination greatly reduces disease, disability, death and inequity worldwide. Bull World Health Organ 2008;86:140-6.

14. Luyten J, Beutels $P$. The social value of vaccination programs: beyond cost-effectiveness. Health Aff 2016;35:212-8.

15. Moher $D$, Shamseer $L$, Clarke $M$, et al. Preferred reporting items for systematic review and meta-analysis protocols (PRISMA-P) 2015 statement. Syst Rev 2015;4:1.

16. Shamseer L, Moher D, Clarke M, et al. Preferred reporting items for systematic review and meta-analysis protocols (PRISMA-P) 2015: elaboration and explanation. BMJ 2015;349:97647.

17. World Bank. World Bank country and lending groups historical classification by country. https://datahelpdesk.worldbank.org/ knowledgebase/articles/906519 (accessed 21 Jul 2016).

18. Moher D, Liberati A, Tetzlaff J, et al. Preferred reporting items for systematic reviews and meta-analyses: the PRISMA statement. Ann Intern Med 2009;151:264-9.

19. Wells GA, Shea B, O'Connell D, et al. The Newcastle-Ottawa Scale (NOS) for assessing the quality of nonrandomised studies in metaanalyses, 2000.

20. Anon. Newcastle-Ottawa Scale adapted for cross-sectional studies. https://www.biomedcentral.com/content/supplementary/1471-245813-154-S3.doc (accessed 22 Aug 2016).

21. Pluye P, Robert E, Cargo M, et al. Proposal: a mixed methods appraisal tool for systematic mixed studies reviews, $2011 \mathrm{http} / / /$ mixedmethodsappraisaltoolpublic.pbworks.com. Archived by WebCite $®$ at. http://www.webcitation.org/5tTRTc9yJ (accessed 05 Sep 2016)

22. Liberati A, Altman DG, Tetzlaff J, et al. The PRISMA Statement for Reporting Systematic Reviews and Meta-Analyses of Studies that Evaluate Health Care Interventions: explanation and elaboration. Ann Intern Med 2009;151:W65. 